Original article

\title{
Synthesis, characterization, antimicrobial and single crystal X-ray crystallographic studies of some new sulfonyl, 4-chloro phenoxy benzene and dibenzoazepine substituted benzamides
}

\author{
B.S. Priya ${ }^{\text {a }}$, S. Nanjunda Swamy ${ }^{\text {a }}$, M.V. Tejesvi ${ }^{b}$, Basappa ${ }^{\text {a }}$, G. Sarala ${ }^{\text {c }}$, S.L. Gaonkar ${ }^{\text {a }}$, \\ S. Naveen ${ }^{c}$, J. Shashidhara Prasad ${ }^{c}$, K.S. Rangappa ${ }^{a, *}$ \\ ${ }^{a}$ Department of Studies in Chemistry, University of Mysore, Manasagangotri, Mysore-570 006, Karnataka, India \\ ${ }^{\mathrm{b}}$ Department of Studies in Biotechnology, University of Mysore, Manasagangotri, Mysore-570 006, Karnataka, India \\ ${ }^{\mathrm{c}}$ Department of Studies in Physics, University of Mysore, Manasagangotri, Mysore-570 006, Karnataka, India
}

Received in revised form 18 April 2006; accepted 29 May 2006

Available online 05 July 2006

\begin{abstract}
A new class of benzamide derivatives $\mathbf{3 a}(\mathbf{I}-\mathbf{V I})$ and $\mathbf{3 b}(\mathbf{I}-\mathbf{V I})$, bearing different bioactive moieties were synthesized and evaluated for their efficacy as antimicrobials in vitro. Compounds 3bVI, 3aII, 3aV, 3bIII, 3aVI, 3bII showed significant antibacterial activity and 3bIII, 3bII, 3aIV, 3bV, 3bVI, 3aI exhibit significant antifungal activity. The title compounds are characterized by spectral and elemental analysis. Compounds 2-methoxy- $N$-[4-(thiazol-2-yl-sulfamoyl)-phenyl]-benzamide 3aII and 2-(2-(2-ethoxybenzoylamino) phenethyl)- $N$-(2-ethoxybenzoyl) benzenamine $\mathbf{3 b V}$ are characterized by the single crystal X-ray studies. Compound 3aII crystallizes in monoclinic space group $\mathrm{P} 2{ }_{1}$ and $\mathbf{3 b V}$ in triclinic space group $\boldsymbol{P}$-1. Compounds $\mathbf{3 a I I}$ and $\mathbf{3 b V}$ exhibit both inter and intra molecular hydrogen bonding.
\end{abstract}

(C) 2006 Elsevier Masson SAS. All rights reserved.

Keywords: Benzamide; Antibacterial; Antifungal; Monoclinic; Triclinic

\section{Introduction}

The need for new antimicrobial agents is greater than ever because of the emergence of multidrug resistance in common pathogens, the rapid emergence of new infections, and the potential for use of multidrug-resistant agents in bioweapons. Solutions encouraging and facilitating the development of new antimicrobial agents are needed [1]. Compound comprising of an amide bond backbone have a wide range of biological activities. Thus among the natural and synthetic substituted amide derivatives, there are compounds possessing anti-proliferative [2], antiviral, antimalarial, general anesthetics [3], anti-inflam-

\footnotetext{
${ }^{*}$ Corresponding author

E-mail addresses:
}

rangappaks@chemistry.uni-mysore.ac.in, rangappaks@yahoo.com (K.S. Rangappa). matory [4], antimicrobials [5] and in treatment of Alzheimer's diseases [6]. From the standpoint of elaborated complex natural product and process chemistry, we have targeted in synthesizing novel amide derivatives, which plays an extremely important role in therapeutic as well as in medicinal field. One of the important categories of medicine is antibiotic, where sulfonamides are used for the treatment of bacterial infections [7], in which the amide moiety may exert their effects by modifying the metabolic activity of the invaded pathogenic microorganisms. These categories of medicines on absorption into the system interfere with microbe's growth or kill them. Penicillin, erythromycin, tetracycline, norfloxacin, etc. come under this category for the treatment of antimicrobials. Our previous studies on the synthesis of heterocycles like isoxazolines, novel isoxazolidines, 1,2-benzisoxazole substituted amides and chroman-2-carboxamides showed a wide spectrum of antimicrobial activities [8-12]. In connection with our efforts, in synthesizing and identifying the various biological targets, herein we 
report the synthesis of new class of substituted benzamides and their antimicrobial activity in vitro against different strains.

\section{Chemistry}

Amide moiety is versatile in organic compounds since all the three atoms in the $\mathrm{O}-\mathrm{C}-\mathrm{N}$ chain are potentially reactive. Several efficient methods have been exploited for the amidation using specific dehydrating reagents under mild liquid-phase condition. The synthesis of substituted amides began with the synthesis of various acid chlorides, where the acid chloride undergoes condensation reaction with different amines in presence of triethylamine as acid scavenger in dichloroethane as solvent. The physical data of newly synthesized molecules is shown in Table 1 and the synthetic scheme shown in Schemes 1, 2 and 3.
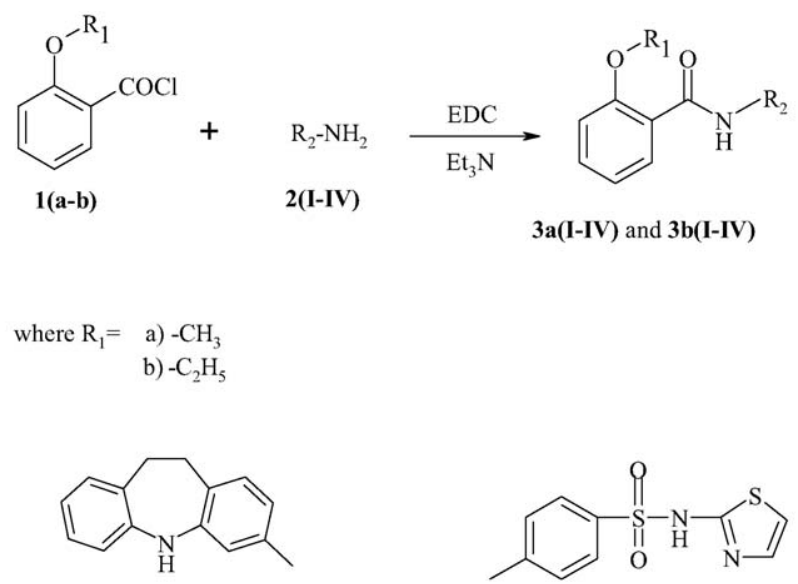

$\mathrm{R}_{2}=$

I

II<smiles>Cc1ccc(S(=O)(=O)Nc2nccc(C)n2)cc1</smiles>

III<smiles>Cc1ccc(S(=O)(=O)Nc2ncccn2)cc1</smiles>

IV<smiles>Nc1ccccc1CCc1ccccc1N</smiles>

$2 \mathrm{~V}$<smiles>Nc1ccccc1Oc1ccc(Cl)cc1N</smiles>

2 VI

$\mathrm{EDC}=$ ethylene dichloride<smiles>[R]Oc1ccccc1C(=O)Nc1ccccc1CCc1ccccc1NC(=O)c1ccccc1O[R]</smiles>

3a V and $\mathbf{3 b V}$
Scheme 2

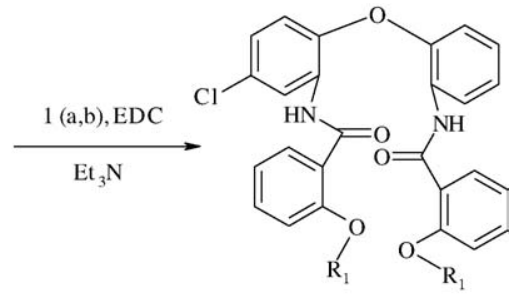

3a VI and 3b VI
Scheme 3

\section{Results and discussion}

\subsection{Chemistry}

Series of benzamides using different acid chlorides and amines were obtained in very good yield in the ratio of 70 $85 \%$ with a purity of $90-95 \%$. IR spectrum of the synthesized compounds showed $\mathrm{N}-\mathrm{H}$ bond stretching in the region of $3300-3400 \mathrm{~cm}^{-1}$ and the $\mathrm{C}=\mathrm{O}$ bond stretching in the region of $1600-1700 \mathrm{~cm}^{-1}$. Synthesized compounds were characterized by spectral, elemental analysis and finally by single crystal $\mathrm{X}$-ray studies for the compounds $\mathbf{3 a I I}$ and $\mathbf{3 b V}$.

\subsection{Crystal structure analysis of $\mathbf{3 a I I}$ and $\mathbf{3 b} \mathrm{V}$}

Figs. 1 and 2 represent the ORTEP of the molecules 3aII and $3 \mathbf{b V}$ with thermal ellipsoids at $50 \%$ probability with selected bond lengths and bond angles. Table 2 represents crystal and experimental data of the molecules $3 \mathbf{a I I}$ and $\mathbf{3 b V}$. Compound 3aII exhibits both intramolecular and intermolecu-

Table 1

Reaction condition and physical data of benzamide series

\begin{tabular}{llllll}
\hline Amides & Time $(\mathrm{h})$ & $R_{\mathrm{f}}$ value & Eluent & Yield $(\%)$ & m.p. $\left({ }^{\circ} \mathrm{C}\right)$ \\
\hline 3a I & 4 & 0.72 & Chloroform/methanol $9: 1$ & 75 & Oily \\
3a II & 4 & 0.63 & Chloroform/methanol $9: 1$ & 75 & $179-182$ \\
3a III & 5 & 0.54 & Chloroform/methanol $9: 1$ & 80 & Oily \\
3a IV & 6 & 0.66 & Chloroform/methanol $9: 1$ & 85 & Oily \\
3a V & 4 & 0.78 & Chloroform/methanol $9: 1$ & 80 & Oily \\
3a VI & 4 & 0.64 & Chloroform/methanol $9: 1$ & 70 & Oily \\
3b I & 4 & 0.55 & Benzene/ethyl acetate $9: 1$ & 75 & $205-210$ \\
3b II & 5 & 0.82 & Benzene/ethyl acetate $9: 1$ & 70 & $215-217$ \\
3b III & 6 & 0.64 & Benzene/ethyl acetate $9: 1$ & 70 & $224-228$ \\
3b IV & 4 & 0.67 & Benzene/ethyl acetate $9: 1$ & 70 & $117-120$ \\
3b V & 4 & 0.58 & Chloroform/methanol $9: 1$ & 85 & 95 \\
3b VI & 4 & 0.75 & & & $98-102$ \\
\hline
\end{tabular}




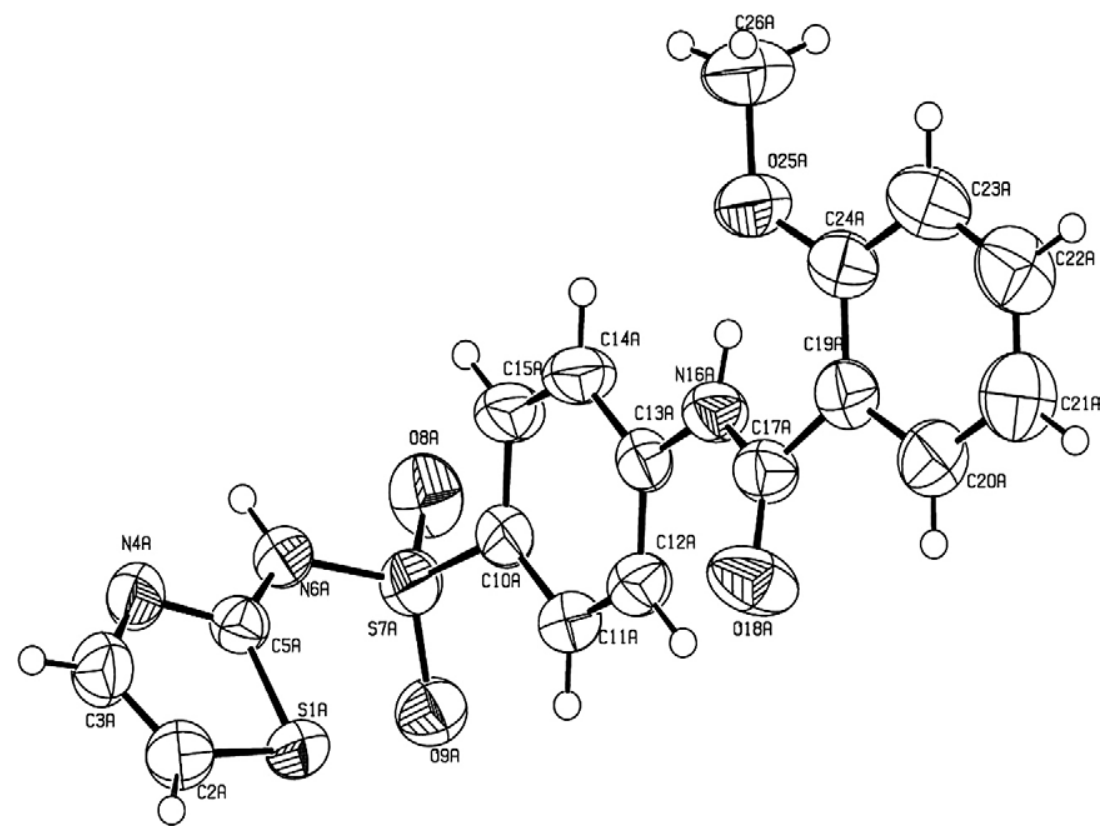

Fig. 1. ORTEP diagram of molecule 3aII at 50\% Probability.

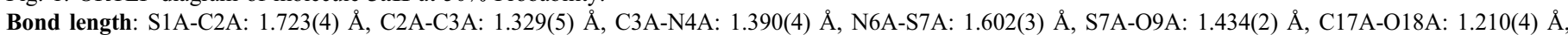

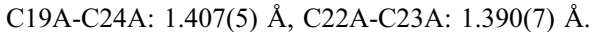

Bond angles: O8A-S7A-N6A: 104.80(2) ${ }^{\circ}$, O9A-S7A-C10A: 107.61(2) ${ }^{\circ}$ N6A-S7A-C10A: 105.15(2) ${ }^{\circ}$, C17A-N16A-C13A: 129.0(3) ${ }^{\circ}$, O18A-C17A-N16A: 122.6 $(3)^{\circ}$, O25A-C24A-C23A: $123.3(3)^{\circ}, \mathrm{C} 24 \mathrm{~A}-\mathrm{O} 25 \mathrm{~A}-\mathrm{C} 26 \mathrm{~A}: 119.5(3)^{\circ}$.

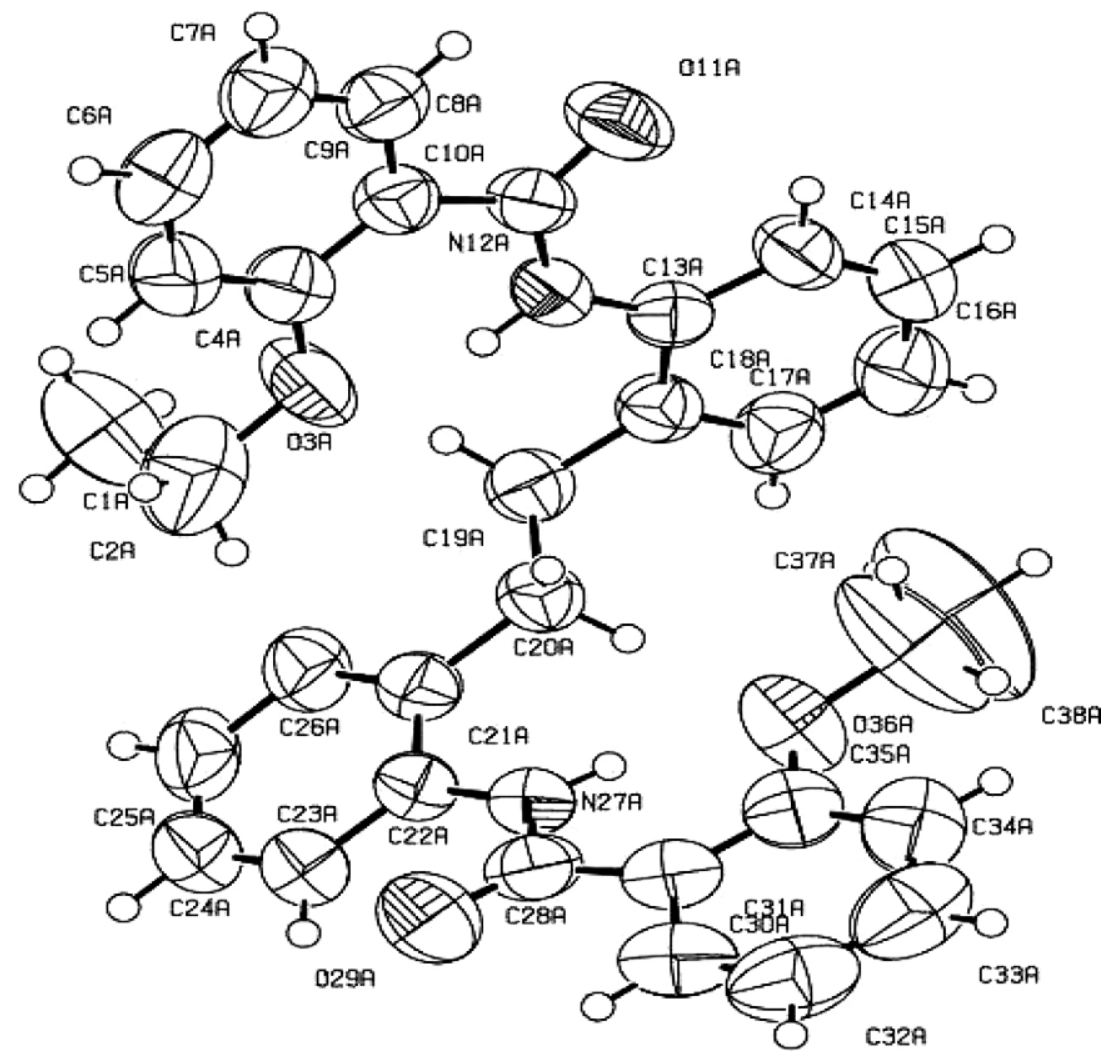

Fig. 2. ORTEP diagram of molecule $3 \mathbf{b V}$ at $50 \%$ Probability.

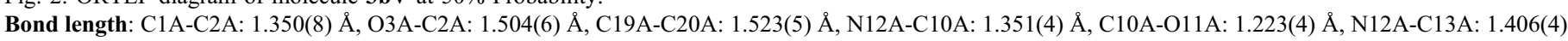

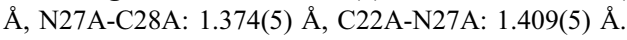

Bond angles: C1A-C2A-O3A:103.5(6) ${ }^{\circ}$ C9A-C10A-O11A: 119.6(3) $)^{\circ}$ O11A-C10A-N12A: $122.3(3)^{\circ}$, N12A-C13A-C14A: 123.3(3) ${ }^{\circ}$, N12A-C13A-C18A: 117.8 $(3)^{\circ}$, C18A-C19A-C20A: 111.0(3) ${ }^{\circ}$, N27A-C28A-O29A: 122.3(4) ${ }^{\circ}$, C22A-N27A-C28A: 126.9 (3) . 
Table 2

Crystal data and structure refinement for $\mathbf{3 a I I}$ and $\mathbf{3 b V}$

\begin{tabular}{|c|c|c|}
\hline Identification code & 3aII & $\mathbf{3 b V}$ \\
\hline$\overline{\mathrm{CCDC}}$ number & 293717 & 293718 \\
\hline Formula weight & 389.44 & 508.24 \\
\hline Temperature & $293(2) \mathrm{K}$ & $293(2) \mathrm{K}$ \\
\hline Reflections for cell determination & 7270 & 4657 \\
\hline Wavelength & $0.71073 \AA$ & $0.71073 \AA$ \\
\hline Crystal system & Monoclinic & Triclinic \\
\hline \multirow{2}{*}{ Cell dimensions } & $c=15.284(1) \AA, \alpha=90^{\circ}$, & $c=17.447(3) \AA, \alpha=88.402(3) \AA$ \\
\hline & $\beta=90.802(4)^{\circ}, \gamma=90^{\circ}$ & $\beta=75.728(8) \AA, \gamma=81.582(8) \AA$ \\
\hline Volume & $1787.5(2) \AA^{3}$ & $2674.6(7) \AA^{3}$ \\
\hline$Z$ & 4 & 4 \\
\hline Density (calculated) & $1.447 \mathrm{mg} \mathrm{m}^{-3}$ & $1.261 \mathrm{mg} \mathrm{m}^{-3}$ \\
\hline & $-23 \leq l \leq 23$ & $-23 \leq l \leq 26$ \\
\hline Reflections collected & 10874 & 17274 \\
\hline Independent reflections & $10874[R($ int $)=0.0000]$ & $11695[R$ (int $)=0.0375]$ \\
\hline Refinement method & Full-matrix least-squares on $F^{2}$ & Full-matrix least-squares on $F^{2}$ \\
\hline Data/restraints/parameters & $10874 / 1 / 472$ & $11695 / 0 / 690$ \\
\hline Goodness-of-fit on $F^{2}$ & 1.092 & 1.332 \\
\hline Final $\mathrm{R}$ indices $[I>2 \sigma(I)]$ & $R 1=0.0532, \omega R 2=0.1360$ & $R 1=0.0962, \omega R 2=0.2465$ \\
\hline$R$ indices (all data) & $R 1=0.0862, \omega R 2=0.1707$ & $R 1=0.2013, \omega R 2=0.2965$ \\
\hline Extinction coefficient & $0.0100(18)$ & $0.0064(18)$ \\
\hline Largest diff. peak and hole & $0.516 \mathrm{e}^{-3}$ and $-0.306 \mathrm{e} \AA^{-3}$ & $0.396 \mathrm{e}^{-3}$ and $-0.273 \mathrm{e}^{-3}$ \\
\hline
\end{tabular}

lar hydrogen bonding. The intermolecular bonds are between N6A-H6A-N4B with bond length 2.863(4) $\AA$, bond angle $143^{\circ}$ and symmetry $\{x,-1+y, z\}$, the N6B-H6B-N4A with bond length $2.926(4) \AA$, bond angle $144^{\circ}$ and symmetry $\{x$, $1+\mathrm{y}, \mathrm{z}\}$, the $\mathrm{C} 2 \mathrm{~A}-\mathrm{H} 2 \mathrm{~A}-\mathrm{O} 18 \mathrm{~A}$ with bond length $3.047(5) \AA$, bond angle $116^{\circ}$ and symmetry $\{2-\mathrm{x},-1 / 2+\mathrm{y},-\mathrm{z}\}$ and C15A-H15A-O9A with bond length 3.454(4) $\AA$, bond angle $158^{\circ}$ and symmetry $\{2-\mathrm{x},-1 / 2+\mathrm{y},-\mathrm{z}\}$.
Compound $\mathbf{3 b V}$ exhibits both intramolecular and intermolecular hydrogen bonding. The intermolecular bonds are between C2B-H2B-O11A with bond length 3.304(5) $\AA$, bond angle $132^{\circ}$ and symmetry $\{1-\mathrm{x}, 1-\mathrm{y}, 1-\mathrm{z}\}$, the C1A-H1A$\mathrm{O} 11 \mathrm{~B}$ with bond length $3.385(6) \AA$, bond angle $150^{\circ}$ and symmetry $\{1-\mathrm{x}, 1-\mathrm{y},-\mathrm{z}\}$, and the C37B-H37B-O29A with bond length $3.395(1) \AA$, bond angle $140^{\circ}$ and symmetry $\{1-$ $\mathrm{X},-\mathrm{Y},-\mathrm{Z}\}$, respectively.

Table 3

Minimal inhibitory concentration (MIC) in $\mu \mathrm{g} \mathrm{ml}^{-1}$ of compounds against tested bacterial strains by microdilution method

\begin{tabular}{llllll}
\hline Compound & \multicolumn{5}{c}{ Minimal inhibitory concentration (MIC) in $\mu \mathrm{g} \mathrm{ml}{ }^{-1}$} \\
\cline { 2 - 5 } & Bacillus substilis & Escherichia coli & Pseudomonas fluorescens & Xanthomonas campestris pvs. & Xanthomonas oryzae \\
\hline 3a I & $32 \pm 1.2$ & $28 \pm 1.2$ & $25 \pm 1.1$ & $24 \pm 1$ & $25 \pm 1.1$ \\
3a II & $8 \pm 0.32$ & $7 \pm 0.3$ & $6 \pm 0.2$ & $5 \pm 0.2$ & $8 \pm 0.3$ \\
3a III & $23 \pm 1$ & $20 \pm 0.8$ & $16 \pm 0.7$ & $12 \pm 0.3$ & $15 \pm 0.6$ \\
3a IV & $25 \pm 0.9$ & $17 \pm 0.7$ & $17 \pm 0.72$ & $6 \pm 0.2$ & $9 \pm 0.7$ \\
3a V & $9 \pm 0.35$ & $8 \pm 0.3$ & $7 \pm 0.21$ & $12 \pm 0.5$ \\
3a VI & $11 \pm 0.95$ & $10 \pm 0.4$ & $12 \pm 0.4$ & $8 \pm 0.3$ & $24 \pm 1$ \\
3b I & $30 \pm 1.2$ & $29 \pm 1.2$ & $26 \pm 1$ & $23 \pm 1$ & $14 \pm 0.5$ \\
3b II & $18 \pm 0.8$ & $15 \pm 0.6$ & $13 \pm 0.40$ & $7 \pm 0.36$ & $10 \pm 0.3$ \\
3b III & $10 \pm 0.4$ & $9 \pm 0.3$ & $11 \pm 0.4$ & $13 \pm 0.5$ & $16 \pm 0.6$ \\
3b IV & $22 \pm 0.9$ & $18 \pm 0.7$ & $14 \pm 0.6$ & $12 \pm 0.4$ & $15 \pm 0.42$ \\
3b V & $24 \pm 1$ & $19 \pm 0.75$ & $15 \pm 0.62$ & $3 \pm 0.1$ & $6 \pm 0.2$ \\
3b VI & $6 \pm 0.2$ & $18 \pm 0.2$ & $4 \pm 0.13$ & - & - \\
Streptomycin & $23 \pm 0.9$ & - & $15 \pm 0.6$ & $12 \pm 0.42$ & $16 \pm 0.6$ \\
Tetracycline & - & & - &
\end{tabular}

${ }^{a}$ Values are mean of three determinations, the ranges of which are less than $5 \%$ of the mean in all cases. 


\subsection{Biology}

In continuation of our efforts in synthesizing new antimicrobials, we have developed new derivatives of benzamides and tested for their efficacy as antimicrobials in vitro by disk diffusion, microdilution and turbidometric methods against different strains. Nystatin was used as positive control against fungi, streptomycin and tetracycline against bacteria. The tests were repeated thrice and the results are reported as mean of at least three determinations. Antibacterial activity of the compounds tested is shown in Tables 3 and 4. From our results, compounds $\mathbf{3 b V I}$, 3aII, 3aV, $\mathbf{3 b I I I}, \mathbf{3 a V I}$ and $\mathbf{3 b I I}$ showed significant inhibitory activity in the following order 3bVI $>$ 3aII $>$ $\mathbf{3 a V}>\mathbf{3 b I I I}>\mathbf{3 a V I}>\mathbf{3 b I I}$. Compounds $3 \mathrm{bVI}$ and 3aVI bearing the bioactive phenoxy moiety, difference in the activity between $3 \mathrm{bVI}$ and $3 \mathrm{aVI}$ might be attributed to the presence of ethoxy and methoxy group at $\mathrm{R}_{1}$ position, respectively. Compound 3aII showed increased activity compared to $\mathbf{3 b I I}$ both bearing sulfathiazole moiety, the presence of different substituents at $\mathrm{R}_{1}$ makes 3aII active. Compounds $\mathbf{3 a V}$ and 3b III showed good activity bearing different moieties and substituents at $R_{1}$ and $R_{2}$ position, respectively. Compounds 3bIV and 3aIV bearing sulfadiazine moiety at $R_{2}$, ethoxy and methoxy at $R_{1}$, respectively, showed moderate potency. Compounds 3aI and $\mathbf{3 b I}$ were not effective against any of the strains tested. Antifungal activity of the compounds tested is shown in Tables 5 and 6. From our results, compounds 3bIII, 3bII, 3aIV, 3bV, 3bVI and 3aI showed significant inhibitory activity in the following order $\mathbf{3 b I I I}>\mathbf{3 b I I}>\mathbf{3 a I V}>$ $\mathbf{3 b V}>\mathbf{3 b V I}>3 \mathrm{aI}$. Interestingly, we found compounds 3aII, $\mathbf{3 a V}$ and 3aVI showed significant inhibitory activity against the fungal strains tested but they exhibited moderate potency against bacterial strains. Compound 3aI showed good antifungal activity but was ineffective against bacteria. Compounds 3aII, 3aVI, 3bIV and 3aIII exhibited moderate antifungal activity. Compounds $\mathbf{3 b I}$ and $\mathbf{3 a V}$ were not effective against any of the fungus tested.

Table 4

Inhibitory zone (diameter) mm of compounds against tested bacterial strains by disk diffusion method

\begin{tabular}{|c|c|c|c|c|c|}
\hline \multirow[t]{2}{*}{ Compound } & \multicolumn{5}{|c|}{ Inhibitory zone (diameter) $\mathrm{mm}$} \\
\hline & Bacillus substilis & Escherichia coli & Pseudomonas fluorescens & Xanthomonas campestris pvs. & Xanthomonas oryzae \\
\hline 3a I & $6 \pm 0.2$ & $2 \pm 0.06$ & $5 \pm 0.18$ & $2 \pm 0.08$ & $3 \pm 0.08$ \\
\hline 3a II & $30 \pm 1.1$ & $38 \pm 1.7$ & $42 \pm 1.8$ & $28 \pm 1.2$ & $32 \pm 1.2$ \\
\hline 3a III & $14 \pm 0.52$ & $19 \pm 0.7$ & $23 \pm 1$ & $18 \pm 0.8$ & $16 \pm 0.5$ \\
\hline 3a IV & $15 \pm 0.59$ & $20 \pm 0.8$ & $22 \pm 0.9$ & $15 \pm 0.65$ & $17 \pm 0.7$ \\
\hline $3 \mathbf{a ~ V}$ & $28 \pm 1.2$ & $36 \pm 1.5$ & $40 \pm 1.6$ & $25 \pm 1.1$ & $30 \pm 1.1$ \\
\hline 3a VI & $25 \pm 1$ & $30 \pm 1.3$ & $34 \pm 1.5$ & $21 \pm 0.9$ & $25 \pm 0.9$ \\
\hline 3b I & $4 \pm 0.1$ & $3 \pm 0.09$ & $7 \pm 0.28$ & $5 \pm 0.18$ & $4 \pm 0.17$ \\
\hline 3b II & $22 \pm 0.9$ & $25 \pm 1.1$ & $30 \pm 1.2$ & $19 \pm 0.8$ & $20 \pm 0.8$ \\
\hline 3b III & $27 \pm 1.1$ & $32 \pm 1.5$ & $36 \pm 1.5$ & $22 \pm 0.9$ & $27 \pm 1$ \\
\hline 3b IV & $16 \pm 0.7$ & $17 \pm 0.52$ & $20 \pm 0.8$ & $16 \pm 0.6$ & $14 \pm 0.6$ \\
\hline $3 b \mathrm{~V}$ & $17 \pm 0.6$ & $18 \pm 0.8$ & $21 \pm 0.82$ & $17 \pm 0.62$ & $15 \pm 0.62$ \\
\hline 3b VI & $32 \pm 1.2$ & $40 \pm 1.3$ & $45 \pm 2.1$ & $30 \pm 1.1$ & $35 \pm 1.23$ \\
\hline Streptomycin & $15 \pm 0.5$ & $19 \pm 0.7$ & $22 \pm 0.9$ & - & - \\
\hline Tetracycline & - & - & - & $16 \pm 0.61$ & $15 \pm 0.52$ \\
\hline
\end{tabular}

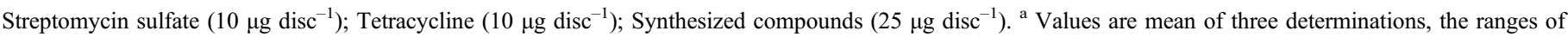
which are less than $5 \%$ of the mean in all cases.

Table 5

Minimal inhibitory concentration (MIC) in $\mu \mathrm{M}$ of compounds against tested fungal strains by Turbidometric method

\begin{tabular}{|c|c|c|c|c|c|}
\hline \multirow[t]{2}{*}{ Compound } & \multicolumn{5}{|c|}{ Minimal inhibitory concentration (MIC) in $\mu \mathrm{M}$} \\
\hline & Aspergillus niger & Aspergillus flavus & Fusarium oxysporum & Trichoderma species & Fusarium moniliforme \\
\hline 3a I & $26 \pm 1.1$ & $30 \pm 1.3$ & $32 \pm 1.2$ & $25 \pm 1.05$ & $27 \pm 1.15$ \\
\hline 3a II & $30 \pm 1.2$ & $34 \pm 1.3$ & $37 \pm 1.4$ & $30 \pm 1.2$ & $33 \pm 1.4$ \\
\hline 3a III & $31 \pm 1.22$ & $36 \pm 1.4$ & $38 \pm 1.6$ & $29 \pm 1.2$ & $35 \pm 1.3$ \\
\hline 3a IV & $17 \pm 0.65$ & $20 \pm 0.8$ & $19 \pm 0.7$ & $17 \pm 0.7$ & $18 \pm 0.7$ \\
\hline $3 \mathbf{a} \mathbf{V}$ & $42 \pm 1.5$ & $47 \pm 1.4$ & $61 \pm 2.5$ & $44 \pm 1.9$ & $47 \pm 1.9$ \\
\hline 3a VI & $31 \pm 1.1$ & $35 \pm 1.5$ & $38 \pm 1.6$ & $29 \pm 1.2$ & $34 \pm 1.4$ \\
\hline 3b I & $48 \pm 2$ & $50 \pm 2.1$ & $52 \pm 2.3$ & $49 \pm 1.9$ & $51 \pm 2.1$ \\
\hline 3b II & $15 \pm 0.6$ & $15 \pm 0.6$ & $16 \pm 0.6$ & $13 \pm 0.5$ & $14 \pm 0.5$ \\
\hline 3b III & $13 \pm 0.5$ & $17 \pm 0.6$ & $18 \pm 0.7$ & $15 \pm 0.55$ & $16 \pm 0.62$ \\
\hline 3b IV & $29 \pm 1.2$ & $36 \pm 1.4$ & $39 \pm 1.7$ & $31 \pm 1.3$ & $35 \pm 1.5$ \\
\hline $3 b \mathrm{~V}$ & $19 \pm 0.67$ & $22 \pm 0.9$ & $21 \pm 0.9$ & $20 \pm 0.8$ & $23 \pm 1.1$ \\
\hline 3b VI & $22 \pm 1$ & $25 \pm 0.98$ & $27 \pm 1.2$ & $22 \pm 0.9$ & $25 \pm 1.5$ \\
\hline Nystatin & $30 \pm 1.2$ & $35 \pm 1.4$ & $37 \pm 1.5$ & $29 \pm 0.75$ & $33 \pm 1.5$ \\
\hline
\end{tabular}

${ }^{\text {a }}$ Values are mean of three determinations, the ranges of which are less than $5 \%$ of the mean in all cases. 
Table 6

Inhibitory zone (diameter) mm of compounds against tested fungal strains by disk diffusion method

\begin{tabular}{|c|c|c|c|c|c|}
\hline \multirow[t]{2}{*}{ Compound } & \multicolumn{5}{|c|}{ Inhibitory zone (diameter) $\mathrm{mm}$} \\
\hline & Aspergillus niger & Aspergillus flavus & Fusarium oxysporum & Trichoderma species & Fusarium moniliforme \\
\hline 3a I & $16 \pm 0.6$ & $17 \pm 0.71$ & $19 \pm 0.7$ & $21 \pm 1$ & $21 \pm 0.9$ \\
\hline 3a II & $13 \pm 0.45$ & $14 \pm 0.58$ & $17 \pm 0.65$ & $18 \pm 0.8$ & $20 \pm 0.8$ \\
\hline 3a III & $11 \pm 0.4$ & $16 \pm 0.7$ & $15 \pm 0.6$ & $17 \pm 0.65$ & $17 \pm 0.67$ \\
\hline 3a IV & $20 \pm 0.8$ & $22 \pm 0.9$ & $24 \pm 1$ & $25 \pm 1.1$ & $28 \pm 1.2$ \\
\hline $3 a \mathbf{V}$ & $4 \pm 0.14$ & $5 \pm 0.2$ & $8 \pm 0.31$ & $2 \pm 0.08$ & $1 \pm 0.02$ \\
\hline 3a VI & $14 \pm 0.6$ & $15 \pm 0.6$ & $18 \pm 0.78$ & $19 \pm 0.84$ & $19 \pm 0.0 .8$ \\
\hline 3b I & $6 \pm 0.21$ & $4 \pm 0.11$ & $2 \pm 0.09$ & $5 \pm 0.19$ & $7 \pm 0.28$ \\
\hline 3b II & $21 \pm 0.9$ & $24 \pm 1$ & $26 \pm 1$ & $27 \pm 1.2$ & $30 \pm 1.2$ \\
\hline 3b III & $24 \pm 1$ & $27 \pm 1.1$ & $29 \pm 1.2$ & $31 \pm 1.3$ & $34 \pm 1.4$ \\
\hline 3b IV & $12 \pm 0.5$ & $15 \pm 0.59$ & $16 \pm 0.68$ & $20 \pm 0.87$ & $18 \pm 0.78$ \\
\hline $3 \mathbf{b} \mathbf{V}$ & $19 \pm 0.8$ & $20 \pm 0.89$ & $22 \pm 0.95$ & $23 \pm 1$ & $25 \pm 1.2$ \\
\hline 3b VI & $17 \pm 0.7$ & $18 \pm 0.72$ & $20 \pm 0.9$ & $21 \pm 0.9$ & $23 \pm 1.1$ \\
\hline Nystatin & $13 \pm 0.55$ & $15 \pm 0.62$ & $17 \pm 0.74$ & $19 \pm 0.8$ & $18 \pm 0.7$ \\
\hline
\end{tabular}

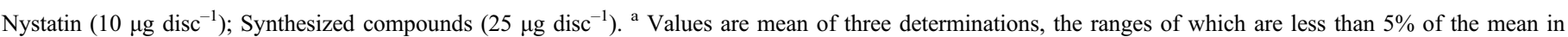
all cases.

\section{Conclusion}

In conclusion, we report the synthesis, antimicrobial studies and crystal structure analysis of newer benzamide derivatives bearing multifunctional moieties such as phenoxy, sulfathiazine, sulfamerazine, sulfadiazine, azepines, etc. Compounds 3bVI, 3aII, 3aV, 3bIII, 3aVI and 3bII showed significant antibacterial activity and 3bIII, 3bII, 3aIV, 3bV, 3bVI and 3aI exhibited antifungal activity against all the strains tested and found to be nonstrain dependent. The antimicrobial activity shown might be partly due to the delocalization of the $\pi$ electrons along the $\mathrm{O}-\mathrm{C}-\mathrm{N}$ chain. This produces partial double-bond character in the $\mathrm{C}(\mathrm{O})-\mathrm{N}$ bond. The versatility of the amide group in forming partial bonds with itself and many other functional groups is partly responsible for the structural subtleties of the biologically important proton derivatives [13, 14]. The crystal data of the compounds $\mathbf{3 a I I}$ and $\mathbf{3 b V}$ reveals that it exhibits both intra and inter molecular hydrogen bonding, which may play a pivotal role for its bioactivities. Modifications to improve the potency of this series by diversification of the position and type of amides are currently under progress in our lab.

\section{Experimental}

\subsection{Chemistry}

The melting points were determined on SELACO-650 hot stage apparatus and are uncorrected. IR (nujol) spectra were measured on Shimadzu 8300 IR spectrophotometer, ${ }^{1} \mathrm{H}$ NMR were recorded on Shimadzu AMX 400-Bruker, 400 MHz spectrometer by using $\mathrm{CDCl}_{3}$ as solvent and TMS as an internal standard (chemical shift in $\delta \mathrm{ppm}$ ). Elemental analyses were obtained on a Vario-EL instrument. TLC was conducted on $0.25 \mathrm{~mm}$ silica gel plates $\left(60 \mathrm{~F}_{254}\right.$, Merck) and Column by silica gel BDH 60-120 mesh. All extracted solvents were dried over $\mathrm{Na}_{2} \mathrm{SO}_{4}$, followed by evaporation in vacuo.

\subsubsection{Substituted amides were synthesized by the reported procedure [12]}

5.1.2. Synthesis of $N-(10,11-$ dihydro-5H-dibenzo[b,f]azepin-3yl)-2-methoxy-benzamide $\mathbf{3 a I}$

It was obtained from the reaction of 10,11-dihydro-5 $\mathrm{H}$ dibenzo[b,f]azepin-3-yl-amine 2I (0.25 g, $1.190 \mathrm{mmol})$, 2methoxybenzoyl chloride $(0.242 \mathrm{~g}, 1.428 \mathrm{mmol})$ and triethylamine $(0.722 \mathrm{~g}, 7.14 \mathrm{mmol})$. The product obtained was oily. IR (cm ${ }^{-1}$ nujol): $3210.8,1675.6,1415.1,1512 .{ }^{1} \mathrm{H} \mathrm{NMR}\left(\mathrm{CDCl}_{3}\right.$, $400 \mathrm{MHz}) \delta: 2.72\left(\mathrm{~s}, 4 \mathrm{H},-\mathrm{CH}_{2}\right) ; 3.12\left(\mathrm{~s}, 3 \mathrm{H},-\mathrm{O}-\mathrm{CH}_{3}\right) ; 3.79$ (s, 1H, NH-IDB); 6.78-7.05 (m, 9H, Ar-H); 7.42 (t, 1H, Ar-H); 7.78 (d, 1H, Ar-H), 10.04 (s, 1H, -NH). Anal. Calcd CHNS: C: 76.72, H: 5.85, N: 8.13. Found: C: 76.66, H: 5.75, N: 8.10.

\subsubsection{Synthesis of 2-methoxy-N-[4-(thiazol-2-yl-sulfamoyl)- phenyl]-benzamide $3 a I I$}

It was obtained from the reaction of 4-amino- $N$-thiazol-2-ylbenzenesulfonamide 2 II $(0.25 \mathrm{~g}, 0.979 \mathrm{mmol})$, 2-methoxybenzoyl chloride $(0.2 \mathrm{~g}, 1.176 \mathrm{mmol})$ and triethylamine $(0.594 \mathrm{~g}$, $5.87 \mathrm{mmol})$. The product obtained was pure white crystalline solid. m.p. $=179-182{ }^{\circ} \mathrm{C} . \mathrm{IR}\left(\mathrm{cm}^{-1}\right.$ nujol): $3345,1700,1478.5$, 1512. ${ }^{1} \mathrm{H}$ NMR $\left(\mathrm{CDCl}_{3}, 400 \mathrm{MHz}\right) \delta: 3.1\left(\mathrm{~s}, 3 \mathrm{H},-\mathrm{O}-\mathrm{CH}_{3}\right)$; $6.61(\mathrm{~d}, 1 \mathrm{H}, \mathrm{Th}-\mathrm{H}) ; 6.90-7.20(\mathrm{~m}, 4 \mathrm{H}, \mathrm{Ar}-\mathrm{H}) ; 7.42-7.79(\mathrm{~m}$, 5H, Ar-H), 10.01 (s, 1H, -CO-NH); 10.40 (s, 1H, - $\left.\mathrm{SO}_{2}-\mathrm{NH}\right)$. Anal. Calcd CHNS: C: 52.43, H: 3.88, N: 10.79, S: 16.47. Found: C: $52.36, \mathrm{H}: 3.72, \mathrm{~N}: 10.71, \mathrm{~S}: 16.42$.

\subsubsection{Synthesis of 2-methoxy-N-[4-(4-methyl-pyrimidin-2-yl- sulfamoyl)-phenyl]-benzamide 3aIII}

It was obtained from the reaction of 4-amino- $N$-(4-methylpyrimidin-2-yl)-benzenesulfonamide $\mathbf{2 I I I} \quad(0.25 \mathrm{~g}, \quad 0.946$ mmol), 2-methoxybenzoyl chloride $(0.192 \mathrm{~g}, 1.129 \mathrm{mmol})$ and triethylamine $(0.574 \mathrm{~g}, 5.672 \mathrm{mmol})$. The product obtained was oily. IR ( $\mathrm{cm}^{-1}$ nujol): $3300.8,1640,1455.7,1523 .{ }^{1} \mathrm{H}$ NMR $\left(\mathrm{CDCl}_{3}, 400 \mathrm{MHz}\right) \delta: 2.50\left(\mathrm{~s}, 3 \mathrm{H}, \mathrm{Ar}-\mathrm{CH}_{3}\right) ; 3.21(\mathrm{~s}$, $\left.3 \mathrm{H},-\mathrm{O}-\mathrm{CH}_{3}\right) ; 6.76(\mathrm{~d}, 1 \mathrm{H}, \mathrm{Py}-\mathrm{H}) ; 6.91-7.01$ (m, 2H, Ar-H); 7.45-7.95 (m, 6H, Ar-H); 8.12 (d, 1H, Py-H); 10.12 (s, 2H, - 
CO-NH); 10.35 (s, 2H, -SO $\left.{ }_{2}-\mathrm{NH}\right)$. Anal. Calcd CHNS: C: $57.27, \mathrm{H}: 4.55$, N: 14.06 , S: 8.05 . Found: C: $57.12, \mathrm{H}: 4.25$, $\mathrm{N}: 13.99, \mathrm{~S}: 7.85$.

\subsubsection{Synthesis of 2-methoxy- $N$-[4-(pyrimidin-2-yl-sulfamoyl)- phenyl]-benzamide $\mathbf{3 a I V}$}

It was obtained from the reaction of 4-amino- $N$-pyrimidin2-yl-benzenesulfonamide 2 IV (0.25 g, $0.998 \mathrm{mmol})$, 2-methoxybenzoyl chloride $(0.203 \mathrm{~g}, 1.194 \mathrm{mmol})$ and triethylamine $(0.605 \mathrm{~g}, 5.988 \mathrm{mmol})$. The product obtained was oily. IR ( $\mathrm{cm}^{-1}$ nujol): $3260,1620,1428,1534.5 .{ }^{1} \mathrm{H}$ NMR $\left(\mathrm{CDCl}_{3}\right.$, $400 \mathrm{MHz}) \delta: 3.16\left(\mathrm{~s}, 3 \mathrm{H},-\mathrm{O}-\mathrm{CH}_{3}\right) ; 6.76(\mathrm{~d}, 1 \mathrm{H}, \mathrm{Py}-\mathrm{H})$; 6.95-7.05 (m, 2H, Ar-H); 7.45-7.95 (m, 6H, Ar-H); 8.12 (t, 2H, Py-H); 10.05 (s, 2H, -CO-NH); 10.29 (s, 2H, - $\mathrm{SO}_{2}-\mathrm{NH}$ ). Anal. Calcd CHNS: C: $56.24, \mathrm{H}: 4.20, \mathrm{~N}: 14.57$, S: 8.34 . Found: C: 56.12, H: 4.17, N: 14.92, S: 8.31 .

\subsubsection{Synthesis of 2-(2-(2-methoxybenzoylamino) phenethyl)- $\mathrm{N}$-(2-methoxybenzoyl) benzenamine $\mathbf{3 a V}$}

It was obtained from the reaction of diamino dibenzyl $\mathbf{2 V}$ ( $0.25 \mathrm{~g}, 1.179 \mathrm{mmol}), 2$-methoxybenzoyl chloride ( $0.4 \mathrm{~g}, 2.353$ $\mathrm{mmol})$ and triethylamine $(0.713 \mathrm{~g}, 7.075 \mathrm{mmol})$. The product obtained was oily. IR ( $\mathrm{cm}^{-1}$ nujol): $3340,1620,1432,1512.6$. ${ }^{1} \mathrm{H} \mathrm{NMR}\left(\mathrm{CDCl}_{3}, 400 \mathrm{MHz}\right) \delta: 2.71\left(\mathrm{~s}, 6 \mathrm{H},-\mathrm{O}-\mathrm{CH}_{3}\right) ; 3.06$ (s, $\left.4 \mathrm{H},-\mathrm{CH}_{2}-\mathrm{Ar}\right) ; 6.92-7.10$ (m, 10H, Ar-H); 7.38 (t, $\left.2 \mathrm{H}, \mathrm{Ar}-\mathrm{H}\right)$; 7.51 (d, 2H, Ar-H), 7.79 (d, 2H, Ar-H); 10.15 (s, 2H, -CONH). Anal. Calcd CHNS: C: 74.50, H: 5.82, N: 5.99. Found: C: $74.425, \mathrm{H}: 5.801, \mathrm{~N}: 5.899$.

\subsubsection{Synthesis of 2-(2-(2-methoxybenzoylamino)-4- chlorophenoxy)-N-(2-methoxybenzoyl)benzamine 3aVI}

It was obtained from the reaction of 2-(2-amino-4-chlorophenoxy) benzenamine 2 VI $(0.25 \mathrm{~g}, 1.21 \mathrm{mmol})$, 2-methoxybenzoyl chloride $(0.288 \mathrm{~g}, 1.694 \mathrm{mmol})$ and triethylamine $(0.734 \mathrm{~g}, 7.26 \mathrm{mmol})$. The product obtained was oily. IR ( $\mathrm{cm}^{-1}$ nujol): $3320,1645,1422,1499.5 .{ }^{1} \mathrm{H}$ NMR $\left(\mathrm{CDCl}_{3}\right.$, $400 \mathrm{MHz}) \delta: 3.06$ (s, 6H, -O-CH $\left.{ }_{3}\right) ; 6.86-6.96$ (m, 5H, Ar-H); 7.05-7.24 (m, 4H, Ar-H); 7.39 (t, 2H, Ar-H); 7.79-7.88 (d, $4 \mathrm{H}$, Ar-H); 10.44 (s, 2H, -NH). Anal. Calcd CHNS: C: 66.87, H: 4.61, N: 5.57. Found: C: 66.77, H: 4.55, N: 5.32.

\subsubsection{Synthesis of N-(10,11-dihydro-5H-dibenzo[b,f]azepin-3- yl)-2-ethoxy-benzamide $3 \boldsymbol{b} \boldsymbol{I}$}

It was obtained from the reaction of 10, 11-dihydro-5 $\mathrm{H}$ dibenzo[b,f]azepin-3-yl-amine 2I (0.25 g, $1.190 \mathrm{mmol}), 2-$ ethoxybenzoyl chloride $(0.263 \mathrm{~g}, 1.424 \mathrm{mmol})$ and triethylamine $(0.722 \mathrm{~g}, 7.14 \mathrm{mmol})$. The product obtained was pale brown solid. m.p. $=130-133{ }^{\circ} \mathrm{C} . \mathrm{IR}\left(\mathrm{cm}^{-1}\right.$ nujol): 3410 , 1650.4, 1455.9, 1489.5. ${ }^{1} \mathrm{H}$ NMR $\left(\mathrm{CDCl}_{3}, 400 \mathrm{MHz}\right) \delta: 1.28$ (t, $\left.3 \mathrm{H},-\mathrm{CH}_{3}\right) ; 2.79$ (s, $\left.4 \mathrm{H},-\mathrm{CH}_{2}\right) ; 3.08$ (q, $\left.2 \mathrm{H},-\mathrm{O}^{-} \mathrm{CH}_{2}-\right) ; 3.82$ (s, 1H, NH-IDB); 6.82-7.06 (m, 9H, Ar-H); 7.44 (t, 1H, Ar-H); 7.75 (d, 1H, Ar-H), 10.02 (s, 1H, -NH). Anal. Calcd CHNS: C: 77.07, H: 6.19, N: 7.82. Found: C: 76.86, H: 6.11, N: 7.71.
5.1.9. Synthesis of 2-ethoxy-N-[4-(thiazol-2-yl-sulfamoyl)phenyl]-benzamide $\mathbf{3 b I I}$

It was obtained from the reaction of 4-amino- $N$ - thiazol-2yl-benzenesulfonamide 2 II $(0.25 \mathrm{~g}, 0.979 \mathrm{mmol})$, 2-ethoxybenzoyl chloride $(0.216 \mathrm{~g}, 1.176 \mathrm{mmol})$ and triethylamine $(0.594 \mathrm{~g}, 5.87 \mathrm{mmol})$. The product obtained was pure white solid. m.p. $=205-210{ }^{\circ} \mathrm{C}$. IR ( $\mathrm{cm}^{-1}$ nujol): $3320.8,1632.5$, 1455.6, 1457.8. ${ }^{1} \mathrm{H}$ NMR $\left(\mathrm{CDCl}_{3}, 400 \mathrm{MHz}\right) \delta: 1.42$ (t, 3H, $\left.\mathrm{CH}_{3}\right) ; 3.19$ (q, 2H, $\left.-\mathrm{O}-\mathrm{CH}_{2}-\right) ; 6.66$ (d, 1H, Th-H); 6.94-7.16 (m, 4H, Ar-H); 7.38-7.75 (m, 5H, Ar-H), 10.16 (s, 2H, -CO$\mathrm{NH}) ; 10.53$ (s, $2 \mathrm{H},-\mathrm{SO}_{2}-\mathrm{NH}$ ). Anal. Calcd CHNS: C: 53.58, H: 4.25, N: 10.41, S: 15.89 . Found: C: 53.46, H: 4.17, N: 10.33, S: 15.72 .

\subsubsection{Synthesis of 2-ethoxy-N-[4-(4-methyl-pyrimidin-2-yl- sulfamoyl)-phenyl]-benzamide $\mathbf{3 b I I I}$}

It was obtained from the reaction of 4-amino- $\mathrm{N}$-(4-methylpyrimidin-2-yl)-benzenesulfonamide $\quad$ 2III $\quad\left(\begin{array}{llll}0.25 & \text { g, } & 0.946\end{array}\right.$ mmol), 2-ethoxybenzoyl chloride $(0.209 \mathrm{~g}, 1.132 \mathrm{mmol})$ and triethylamine $(0.574 \mathrm{~g}, 5.672 \mathrm{mmol})$. The product obtained was pure white solid. m.p. $=215-217{ }^{\circ} \mathrm{C}$. IR $\left(\mathrm{cm}^{-1}\right.$ nujol): $3365,1620,1425,1523 .{ }^{1} \mathrm{H}$ NMR $\left(\mathrm{CDCl}_{3}, 400 \mathrm{MHz}\right) \delta: 1.39$ (t, $\left.3 \mathrm{H},-\mathrm{CH}_{3}\right) ; 2.51$ (s, $\left.3 \mathrm{H}, \mathrm{Ar}-\mathrm{CH}_{3}\right) ; 3.09$ (q, $2 \mathrm{H},-\mathrm{O}-\mathrm{CH}_{2}-$ ); 6.69 (d, 1H, Py-H); 6.96-7.04 (m, 2H, Ar-H); 7.44-7.91 (m, $6 \mathrm{H}, \mathrm{Ar}-\mathrm{H}) ; 8.05$ (d, 1H, Py-H); 10.05 (s, $1 \mathrm{H},-\mathrm{CO}-\mathrm{NH}) ; 10.56$ (s, $1 \mathrm{H},-\mathrm{SO}_{2}-\mathrm{NH}$ ). Anal. Calcd CHNS: C: $58.24, \mathrm{H}: 4.89, \mathrm{~N}$ : 13.58, S: 7.77. Found: C: 58.14, H: 4.75, N: 13.42, S: 7.65.

\subsubsection{Synthesis of 2-ethoxy- $N$-[4-(pyrimidin-2-yl-sulfamoyl)- phenyl]-benzamide $\mathbf{3} \boldsymbol{b I V}$}

It was obtained from the reaction of 4-amino- $N$-pyrimidin2-yl-benzenesulfonamide $2 \mathbf{I V}(0.25 \mathrm{~g}, 0.998 \mathrm{mmol})$, 2-ethoxybenzoyl chloride $(0.221 \mathrm{~g}, 1.197 \mathrm{mmol})$ and triethylamine $(0.605 \mathrm{~g}, 5.988 \mathrm{mmol})$. The product obtained was pure white crystalline solid. m.p. $=224-228{ }^{\circ} \mathrm{C}$. IR $\left(\mathrm{cm}^{-1}\right.$ nujol): 3300 , 1640, 1456, 1546. ${ }^{1} \mathrm{H}$ NMR $\left(\mathrm{CDCl}_{3}, 400 \mathrm{MHz}\right) \delta: 1.39$ (t, $\left.3 \mathrm{H},-\mathrm{CH}_{3}\right) ; 2.29$ (q, $\left.2 \mathrm{H},-\mathrm{O}-\mathrm{CH}_{2}-\right) ; 6.74$ (t, $\left.1 \mathrm{H}, \mathrm{Py}-\mathrm{H}\right), 6.98$ 7.10 (m, 2H, Ar-H); 7.34 (t, 1H, Ar-H); 7.88-7.94 (m, 5H, ArH); 8.23 (dd, 2H, Py-H), 10.16 (s, 2H, -CO-NH); 10.48 (s, 2H, $\left.-\mathrm{SO}_{2}-\mathrm{NH}\right)$. Anal. Calcd CHNS: C: $57.27, \mathrm{H}: 4.55, \mathrm{~N}: 14.06$, S: 8.05. Found: C: 57.22, H: 4.47, N: 14.00, S: 7.95 .

5.1.12. Synthesis of 2-(2-(2-ethoxybenzoylamino)phenethyl) $\mathrm{N}$ (2-ethoxybenzoyl) benzenamine $\mathbf{3 b V}$

It was obtained from the reaction of diamino dibenzyl $\mathbf{2 V}$ (0.25 g, $1.179 \mathrm{mmol})$, 2-ethoxybenzoyl chloride $(0.435 \mathrm{~g}$, $2.358 \mathrm{mmol})$ and triethylamine $(0.713 \mathrm{~g}, 7.075 \mathrm{mmol})$. The product obtained was pale brown crystalline solid. m.p. = 117-120 ${ }^{\circ} \mathrm{C}$. IR ( $\mathrm{cm}^{-1}$ nujol): $3384,1645,1475,1532 .{ }^{1} \mathrm{H}$ NMR $\left(\mathrm{CDCl}_{3}, 400 \mathrm{MHz}\right) \delta: 1.24$ (t, 6H, - $\left.\mathrm{CH}_{3}\right) ; 3.15(\mathrm{~s}, 4 \mathrm{H},-$ $\left.\mathrm{CH}_{2}-\mathrm{Ar}\right) ; 3.55$ (q, 4H, -O-CH 2 ); 6.95-7.14 (m, 10H, Ar-H); 7.42 (t, 2H, Ar-H); 7.54 (d, 2H, Ar-H), 7.76 (d, 2H, Ar-H); 10.45 (s, 2H, -CO-NH). Anal. Calcd CHNS: C: 75.57, H: 6.34, N: 5.51. Found: C: 75.54, H: 6.72, N: 5.42. 


\subsubsection{Synthesis of 2-(2-(2-ethoxybenzoylamino)-4-} chlorophenoxy)- $N$-(2-ethoxybenzoyl) benzamine $3 \boldsymbol{b V I}$

It was obtained from the reaction of 2-(2-amino-4-chlorophenoxy)benzenamine 2 VI $(0.25 \mathrm{~g}, 1.21 \mathrm{mmol})$, 2-ethoxybenzoyl chloride $(0.312 \mathrm{~g}, 1.694 \mathrm{mmol})$ and triethylamine $(0.734 \mathrm{~g}, 7.26 \mathrm{mmol})$. The product obtained was pale brown crystalline solid. m.p. $=98-102{ }^{\circ} \mathrm{C}$. IR ( $\mathrm{cm}^{-1}$ nujol): 3360 , 1660, 1480, 1210. ${ }^{1} \mathrm{H}$ NMR $\left(\mathrm{CDCl}_{3}, 400 \mathrm{MHz}\right) \delta: 1.54(\mathrm{t}$, $\left.6 \mathrm{H},-\mathrm{CH}_{3}\right), 4.29$ (q, $\left.4 \mathrm{H},-\mathrm{O}-\mathrm{CH}_{2}-\right), 6.79-6.92(\mathrm{~m}, 5 \mathrm{H}, \mathrm{Ar}-\mathrm{H})$; 7.01-7.19 (m, 4H, Ar-H); 7.41 (t, 2H, Ar-H); 7.74-7.85 (d, $4 \mathrm{H}, \mathrm{Ar}-\mathrm{H}) ; 10.45$ (s, 2H, -NH). Anal. Calcd CHNS: C: 66.60, H: 4.99, N: 5.55. Found: C: 66.52, H: 4.65, N: 5.42.

\subsection{Crystal structure analysis of $3 a I I$ and $3 b \mathrm{~V}$}

Single crystals of $\mathbf{3 a I I}$ and $\mathbf{3 b V}$ suitable for X-ray diffraction studies were mounted on a glass fiber. The measurements were made on a DIPLabo Imaging Plate system with graphite monochromated $\mathrm{Mo} K_{\alpha}$ radiation. Thirty-six frames of data were collected using oscillation method. Image processing and data reduction were done using Denzo [15]. The structure was solved using maXus [16]. All the non-hydrogen atoms were revealed in the first map itself [17]. Initially, the fullmatrix least squares refinement for 7270 reflections for 3aII and 4657 reflections for $\mathbf{3 b V}$ with isotropic temperature factors for all the non-hydrogen atoms was carried out [18]. The $R 1$ value of $3 \mathrm{aII}$ is 0.0862 . The $R 1$ value of $\mathbf{3 b V}$ is 0.0938 .

\subsection{Antimicrobial activity}

Bacteria and fungal species used were obtained from Department of Studies in Biotechnology, University of Mysore, India, namely, Bacillus substilis, Escherichia coli, Pseudomonas fluorescens, Xanthomonas campestris pvs, Xanthomonas oryzae, Aspergillus niger, Aspergillus flavus, Fusarium oxysporum, Trichoderma species and Fusarium moniliforme. The bacterial strains were maintained on LB agar medium and the filamentous fungi were maintained on potato dextrose agar (PDA) medium at $28{ }^{\circ} \mathrm{C}$. The disk diffusion method [19] was used to determine antibacterial and antifungal activity of the synthesized compounds. Paper discs with DMSO were used as negative controls. The bacteria were grown in LB broth, centrifuged at 10,000 rpm for $5 \mathrm{~min}$, pellet was dissolved in double distilled and used to inoculate the plates. The inoculum was prepared with the spores derived from 5 to 15 days culture on PDA medium for the filamentous fungi. The mycelia were covered with $10 \mathrm{ml}$ of distilled water and the conidia were scraped using a sterile pipette. The spores were recovered after filtration on sterile absorbent cotton and were resuspended in sterile distilled water. The cell density of each inoculum was adjusted with hemocytometer in order to obtain a final concentration of approximately $10^{4} \mathrm{CFU} \mathrm{m}{ }^{-1}$ and $10^{6}$ spores $\mathrm{ml}^{-1}$ for the bacteria and filamentous fungi, respectively. Nystatin (Himedia) was used as a positive control for fungi and streptomycin and tetracycline for bacteria. Each disk contained $10 \mu \mathrm{g}$ of standard drugs and $25 \mu \mathrm{g}$ synthesized compounds. Plates were first kept at $4{ }^{\circ} \mathrm{C}$ for at least 2 hours to allow the diffusion of chemicals and then incubated at $28{ }^{\circ} \mathrm{C}$. Inhibition zones were measured after 24 hours of incubation for bacteria and after 48 hours of incubation for fungi. The microdilution method [20] was followed to determine the minimum inhibitory concentration (MIC) of all the compounds against bacterial strains. The nutrient liquid medium was used as test media. Tests were performed in 96-well round bottom sterile culture plates. The wells of the microdilution plate were inoculated with $180 \mu \mathrm{l}$ of the culture medium containing a final inoculum of $0.5 \times 2.5 \times 10^{3} \mathrm{CFU} \mathrm{m} l^{-1}$ [21]. All the compounds previously solubilized in DMSO were serially diluted twofolds in the liquid medium and had concentration 640-0. $1 \mu \mathrm{g} \mathrm{ml}{ }^{-1}$. Twenty microliter of each concentration was added to each well containing the culture suspension except the growth control well. The final concentration ranged from 64 to $0.01 \mu \mathrm{g} \mathrm{ml}^{-1}$. Plates were incubated at $35{ }^{\circ} \mathrm{C}$ for 48 hours. Growth was assessed at $494 \mathrm{~nm}$ by measuring the optical density in each well using an enzyme immunoassay multiwell reader (Sigma Diagnostic). Turbidometric method $[22,23]$ was used to check antifungal activity of the compounds at different concentrations using nystatin as the positive control and DMSO as the negative control. To the culture tubes containing $1.9 \mathrm{ml}$ of sterile media, $0.1 \mathrm{ml}$ of the test compound was added at sterile conditions. Fresh inoculum was added to all the tubes including standard and controls with a spore concentration adjusted to $1 \times 10^{6}$ spores $\mathrm{ml}^{-1}$. After incubating all tubes at $37{ }^{\circ} \mathrm{C}$ for 48 hours, absorbance was recorded at $610 \mathrm{~nm}$. Percentage of inhibition was calculated according to the formula

$\%$ Inhibition $=100(P-Q) / \mathrm{P}$

where $P=$ absorbance without test sample and $Q=$ absorbance with test sample. Then the MIC was recorded in $\mu \mathrm{M}$. All determinant tests were performed duplicate and the results were reported as mean of these values.

\section{Acknowledgements}

The authors are grateful to CSIR, DST-FIST and UGC-SAP (Phase I) DRS Programme, New Delhi, Government of India for financial assistance under the projects no: 01(1904)/03/ EMR-I, DV6/15/DST/2005-06, SP/I2/FOO/93 and DV4/375/ 2004-05. We are thankful to NMR Research Institute, Bangalore, for NMR spectral analysis.

\section{References}

[1] B. Spellberg, J.H. Powers, E.P. Brass, L.G. Miller, J.E. Edwards, Clin. Infect. Dis. 38 (9) (2004) 1279-1286.

[2] V. Gududuru, H. Hurch, T. James, Dalton, D. Duane, Miler, Bioorg. Med. Chem. Lett. 14 (2004) 5289-5293.

[3] C. Dollery, Therapeutic Drugs, Churchill Livingstone, Edinburg, UK, 1999 (272-278).

[4] E. Igor, Bylov, V. Maksym, Vasylev, V. Yaroshov, Bilokin, Eur. J. Med. Chem. 34 (1999) 997-1001. 
[5] E. Aki-Sener, K.K. Bingol, O. Temiz-Arpaci, I. Yalcin, N. Altanlar, Farmaco 57 (6) (2002) 451-456.

[6] C.V.C. Prasad, W. Jeffery, Noonan, P. Charles, Sloan, Wai Lau, Shikha Vig, F. Michael, Parker, W. David, Smith, B. Steven, Hansel, T. Craig, Polson, M. Dona, Barten, M. Kevin, Felsenstein, B. Susan, Roberts, Bioorg. Med. Chem. Lett. 14 (2004) 1917-1921.

[7] G. Bertram, Katzung., Basic and Clinical Pharmacology. Sixth edition, University of California, San Francisco, 1995.

[8] K.R. Ravikumar, H. Mallesha, K.S. Rangappa, Synth. Commun. 33 (9) (2003) 1545

[9] K.R. Ravikumar, H. Mallesha, Bassapa, K.S. Rangappa, Eur. J. Med. Chem. 38 (2003) 163.

[10] M.P. Sadashiva, H. Mallesha, N.A. Hitesh, K.S. Rangappa, Bioorg. Med. Chem. 12 (24) (2004) 6389-6395.

[11] Basappa, M.P. Sadashiva, K. Mantelingu, S. Nanjunda Swamy, K.S. Rangappa, Bioorg. Med. Chem. 11 (2003) 4539-4544.

[12] B.S. Priya, Basappa, S. Nanjunda Swamy, Kanchugarkoppal, K.S. Rangappa, Bioorg. Med. Chem. 13 (2005) 2623-2628.

[13] P.G. Wyatt, R.C. Bethell, N. Cammack, D. Chandran, N. Dodic, B. Dumaitre, D. Evans, D.V.S. Green, P.L. Hopewell, D.C. Humber, R.B. Lamont, D.C. Orr, S.J. Plested, D.M. Ryan, S.L. Sollis, R. Storer, G.G. Weingarten, J. Med. Chem. 38 (1995) 1657.
[14] S.D. Barton, W.D. Ollis, First ed, in: C.J. Sutherland (Ed.), Comprehensive Organic Chemistry, Pergamon, Oxford, New York, 1979, p. 3100 (Vol. 2).

[15] Z. Otwinowski, W. Minor, in: C.W. Carter Jr., R.M. Sweet (Eds.), Methods in Enzymology, Academic Press, New York, 1997, pp. 307-326 (276)

[16] S. Mackay, C.J. Gilmore, E. Stewart, N. Shanklannd, K. maXus, Computer program for the solution and refinement of crystal structures, Bruker Nonius, MacScience, Japan and The University of Glasgow, The Netherlands, 1999.

[17] G.M. Sheldrick, SHELXS-97, Program for Crystal Structure Solution, University of Göttingen, Germany, 1997.

[18] G.M. Sheldrick, SHELXL-97, Program for the Refinement of Crystal Structures, University of Göttingen, Germany, 1997.

[19] S. Lemriss, B. Marquet, H. Ginestet, L. Lefeuvre, A. Fassouane, P.J. Boiron, Mycol. Med. 13 (2003) 189-192.

[20] J.R. Zgoda, J.R. Porter, Pharmaceutical Biology. 39 (2001) 221-225.

[21] S. Rifai, A. Fassouane, A. Kijjoa, R. Van Soest, Mar. Drugs. 2 (2004) 147-153.

[22] G. Barbaro, A. Battaglia, A. Dondoni, J. Chem, Soc. Sec B 588 (1970).

[23] B.G. Mullen, R.T. Decory, T.J. Mitchell, D.S. Allen, C.R. Kinsolving, V. St Georgiev, J. Med. Chem. 31 (1988) 2008. 